REVISTA MAD, N³6 (2017), PP. 1-20

\title{
Familia y escuela: Algunas reflexiones sobre la diferenciación interna del sistema de la educación
}

\author{
Family and School: Some Reflections on the Inner Differentiation of Educational \\ System
}

Hartmann Tyrell

(R) Facultad de Sociología, Universidad de Bielefeld, Alemania

\section{Raf Vanderstraeten}

Departamento de Sociología, Universidad de Gante, Bélgica

RESUMEN

De manera tradicional, el análisis del proceso de profesionalización en el sistema de educación se ha enfocado en el rol de los profesores en el contexto escolar. En comparación, mucha menor atención se ha prestado a la educación impartida por parte de la familia y, especialmente, los padres. Este énfasis en la enseñanza y su profesionalización tiene indudablemente que ver con el hecho de que, si bien (casi) todos pueden convertirse en padres, la entrada a la enseñanza debe ser regulada y controlada. Pero omite el hecho de que la profesionalización de la enseñanza no sólo afecta a la educación que tiene lugar en las escuelas, sino que también modifica las expectativas de rol asociadas a los padres y la paternidad, puesto que los profesores no se limitan a actuar in loco parentis. En esta dirección, considérese el impacto que tienen en la vida familiar la organización temporal de la jornada y el año escolar así como tareas u observaciones en el libro de anotaciones o ceremonias institucionales como las reuniones de apoderados, etc. Así, se ha iniciado un proceso de "escolarización de la familia" a partir de los diferentes puntos de contacto entre la familia y la escuela. En este artículo, analizamos cómo tiene lugar esta confrontación entre la "autoridad natural" de los padres y la "autoridad aprendida" de los profesores.

PALABRAS ClAVE: Diferenciación social; Sociología de la educación; Sociología de la familia; Interacción social; Organización social

\section{ABSTRACT}

The focus of professionalization in the system of education has traditionally been on teachers in the school context. Much less attention has been devoted to the parents and family education. This focus on teaching and its professionalization has certainly to do with the fact that (almost) everyone can become parent, but that entry into teaching can be regulated and controlled. But the professionalization of teaching not only affects education in schools. The professionalization of teaching has also modified the role expectations with regard to parents and parenting. Teachers are not just acting in loco parentis. Consider the impact on the family of the temporal organization of the school day and the school year. Consider the impact of home work or (re)marks on report cards. Consider the effects of institutionalized ceremonies, such as parent-teacher consultations in primary and secondary schools, etc. The different interfaces between the family and the school have initiated a process of "schooling the family". In this paper, we analyse how the confrontation between the "natural" authority of the parents and the "learned" authority of the teachers actually takes place.

KEYWORDS: Social differentiation; Sociology of education; Sociology of the family; Social interaction; Social organization

REVISTA MAD - REVISTA DEL MAGÍsTER EN ANÁLISIS SisTÉMICO APLICADO A LA SOCIEDAD ISSN 0718-0527

Facultad de Ciencias Sociales, Departamento de Antropología.

Universidad de Chile

www.revistamad.uchile.cl 


\section{INTRODUCCIÓN}

Si se presta atención a la evolución histórica de la educación en Occidente, llama la atención inmediatamente su rápida expansión desde mediados del siglo XVIII. La escuela se convirtió en un breve espacio de tiempo en una parada obligada en el proceso educativo de casi cada niño. El proceso educativo se repartía en (al menos) dos ambientes diferentes: familia y escuela. Esta diferenciación interna ${ }^{1}$ del sistema de la educación ${ }^{2}$ en familia y escuela ha marcado el desarrollo de la identidad de cada niño desde entonces. En la segunda mitad del siglo XVIII ocurrieron cambios fundamentales en este ámbito, los que en pocas décadas transformaron realidades y conciencias. Entonces se tomaron decisiones que repercuten en muchos aspectos hasta hoy en día. En esta dirección:

La diferenciación entre familia y escuela -tanto como ruptura en la biografía como simultaneidad en la vida- es la primera diferenciación de diferencias, la primera diferenciación de sistemas a la que se ve expuesto el niño. Ella debe tener efectos incalculables cuando hoy todos buscan su identidad de esta forma y no de otra (Luhmann \& Schorr 1982: 246ss).

La transformación histórica de la educación forma parte de un profundo proceso de transformación social. Es parte de la transición hacia una formación social caracterizada por la diferenciación funcional. La diferenciación incipiente en el siglo XVIII de un sistema de la educación para toda la población se corresponde inicialmente con los requerimientos y aportes presentados, en particular, en los ámbitos de la política y de la economía. En relación con lo anterior, frecuentemente se enfatiza que el establecimiento de los sistemas nacionales de enseñanza es parte de un proceso más amplio, donde familias y hogares transfieren o pierden algunas de sus tareas o funciones tradicionales ante organizaciones funcionalmente especializadas (Inkeles \& Sirowy 1983).

Sin embargo, también se puede afirmar que, sólo como consecuencia de la pérdida de ciertas funciones (económicas y políticas, entre otras), la familia ha podido encontrar su función o funciones propias -y que justamente por ello ha también aumentado su importancia social. En este sentido, Talcott Parsons (1955: 9ss) considera que la sociedad moderna se

\footnotetext{
${ }^{1}$ Nota del traductor [en adelante: NT]: En adelante traduzco Ausdifferenzierung como diferenciación e Innendifferenzierung como diferenciación interna. Sigo en este sentido la traducción tradicional de los conceptos luhmannianos al español p.ej. Corsi, G., Esposito, E. y Baraldi, C. (1996). Glosario sobre la teoría social de Niklas Luhmann (traducción de Miguel Romero Pérez y Carlos Villalobos, bajo la dirección de Javier Torres Nafarrate). México: Universidad Iberoamericana.

2 NT: En adelante traduzco Erziehungssystem como "sistema de la educación" y no como "sistema educativo" para seguir la idea de Luhmann de que en el sistema de la educación, además de los procesos de enseñanza basados en los sistemas de interacción (Unterrichtssystemen), se incluye también la reflexión sobre asuntos pedagógicos (pädagogischer Reflexion). Esta distinción es relevante también en las reflexiones de los autores, pues ellas consideran las situaciones escolares y la educación familiar como parte del sistema de la educación. Sobre esta terminología, véase Runge, A. \& Muñoz, D. (2005). "Luhmann y el déficit tecnológico en educación: ¿es posible la autorreferencia en el sistema educativo?", Uni-pluri/versidad, 5(3), p. 33.
} 
Familia y escuela: Algunas reflexiones sobre la diferenciación interna del sistema de la educación

caracteriza por una estructura "in which the family is more specialized tan before, but not in any general sense less important, because the society is dependent more exclusively on it for the performance of certain of its vital functions". En la sociedad moderna, la familia se vuelve sensible a la pedagogía. ${ }^{3}$ Aumenta la importancia de la escuela en el proceso educativo, pero no a costa de la familia, pues también aumenta su importancia en este proceso. Ello sugiere la idea de un aumento de las relaciones entre ambos sistemas y provee, en nuestra opinión, de un interesante punto de partida para el análisis de la diferenciación interna del sistema de la educación.

En este artículo nos concentraremos en la diferenciación de la educación escolar y sus efectos en el desarrollo de las identidades de niños y jóvenes. Dado este interés, el foco se ubica en la relación entre familia y escuela. El análisis se dividirá en tres puntos: en primer lugar, se señalan algunas características de la semántica pedagógica del siglo XVIII, la que remarca especialmente la importancia de la educación familiar. A continuación, se explica un cambio de interés: la escuela y la educación escolar se vuelven el centro de la atención con claridad en la segunda mitad del siglo XVIII. Para finalizar, se exponen las distintas dimensiones de la diferenciación entre familia y escuela. En términos generales, este artículo tiene ambiciones exploratorias. En lugar de presentar los factores causales que determinan la relación entre familia y escuela en el sistema de la educación, nos interesa agudizar las posibilidades de observación del fenómeno.

\section{LA PREFERENCIA POR LA EDUCACIÓN FAMILIAR}

En el contexto educativo, la escuela y la familia se vieron frecuentemente enfrentadas. La tesis de que la educación familiar representa una amenaza para la familia fue fundamentada usualmente con argumentos de diversa indole. Así, la escuela socavaría las responsabilidades 'naturales' de los padres al apropiarse de tareas que pertenecen 'realmente' a estos, dañaría también la influencia de los padres, los valores y normas paternos al favorecer la influencia del maestro de escuela, quien es distinto y probablemente contrasta fuertemente con la influencia de los padres. Además, se consideró a la escuela como una fuente potencial de corrupción moral. Los padres tendrían poco o ningún control sobre las personas con las cuales sus hijos tienen contacto en la escuela. Para los padres, el peligro es

\footnotetext{
3 Talcott Parsons llama "socialización" a esta función de la familia. De acuerdo con Parsons, la socialización desempeña la función de asegurar la reproducción continua de las relaciones sociales para el sistema social de la sociedad. La educación puede entonces definirse como socialización intencionada. Ella cumple la misma función de la familia pero bajo condiciones más exigentes que demandan una intencionalidad del proceso de socialización en términos de enseñanza y aprendizaje. Si se elige "socialización" como concepto básico, adquiere entonces importancia especial el rol de la familia para el desarrollo de la identidad de niños y jovenes. No por casualidad Parsons y Bales (1955) publican sus reflexiones bajo el título "Family, Socialization and the Interaction Process". En cambio, si se elige "educación", recibe más atención la escuela.
} 
que sus hijos hicieran malos amigos o se acercaran a las personas equivocadas. En los así llamados 'mejores círculos', p.ej. en Inglaterra, los niños eran enviados a escuelas privadas donde recibían instrucciones detalladas acerca de los compañeros que podían o no frecuentar. Si no era posible asegurar la moral de los niños, entonces al menos se debía intentar mantener el acento al hablar o la forma de presentación personal característicos de los 'mejores círculos'.

A menudo la escuela se consideró una amenaza real para la familia -tan real que incluso influyentes reformadores sociales se manifestaron varias veces contra la difusión de escuelas e instituciones de enseñanza. Dicha oposición se basó en la idea de que la escuela no debía socavar la familia. Las contribuciones que los miembros de la familia podían proporcionarse entre sí, por inadecuadas que fueran, no debían ser entregadas a trabajadores a sueldo y 'sin alma'. A este argumento de la 'santidad de la familia' se agregó después un argumento psicológico. Se afirmó (y aún se afirma) que las guarderías, los hogares y las escuelas, especialmente en lo que respecta a los niños pequeños, eran una amenaza tanto para la relación natural entre padres e hijos como para el desarrollo de los niños. En las publicaciones de John Bowlby se da gran valor al attachment, esto es, la seguridad, confianza y calidez de la relación de los adultos con el niño. La idea es que los inevitables contrastes entre los sistemas de reglas de los distintos ambientes sociales en los cuales los niños crecen amenazarían el correcto desarrollo psíquico del niño. Así opina también, por ejemplo, el pedagogo holandés J.D. Imelman

Algunas veces los jardines infantiles tienen la ambición de distinguirse. Esta ambición puede encontrar su expresión en el deseo de sus encargados de seguir su propio rumbo, eventualmente contra aquel señalado por los padres. Es obvio que esto sería lo último que debiese hacer un jardín infantil normal bajo condiciones normales. No debemos ensuciar nuestras manos más de lo que ya lo están (Imelman 1998: 146).

En cierto modo, se trata de concepciones que habían sido expresadas hace ya mucho tiempo. Mucho antes de encontrar apoyo en John Bowlby, se protestaba contra la expansión de los sistemas de enseñanza. En la primera mitad del siglo XIX, los defensores de la educación preescolar argumentaban que estas instituciones, si es que estuviesen extendidas por todo el territorio, no corromperían la nación ni 'liberarían' a los padres de sus deberes. En este contexto, Samuel Wilderspin, uno de los más conocidos defensores de la difusión de este tipo de educación en Inglaterra, formuló preguntas retóricas en su The Infant System: "Where are the natural guardians of the child? Where are its parents? Are we to encourage their neglect of duty by becoming their substitutes?" (Wilderspin 1840: Cap. 3). Su respuesta fue la siguiente: no se trata de la oposición entre familia y escuela. El peligro para el desarrollo del niño viene de otra parte, de los otros ambientes con los cuales puede entrar en contacto, sobre todo la ca- 
Familia y escuela: Algunas reflexiones sobre la diferenciación interna del sistema de la educación

lle y la delincuencia y las drogas (alcohol, etc.). Esta falta de entusiasmo por el jardín infantil se refleja también en el juicio extremadamente reservado de Sir James Kay-Shuttleworth, conocido como uno de los más importantes pioneros en la instalación del sistema escolar inglés. En una descripción histórica acerca de las infant schools inglesas, las que habían sido pensadas para niños de 2 o 3 hasta 5 años, se acentúa el éxito de estas escuelas, pero se manifiesta también y al mismo tiempo la esperanza que en el futuro dichas instituciones ya no serán necesarias -es decir, cuando "parents more lettered and less sensual, will be less prone to neglect infants and children of a riper age" (Kay-Shuttleworth 1862/1993: 132).

Durante los siglos XVIII y XIX el debate en Inglaterra no se limitó a la educación preescolar, sino que abarcó al sistema de enseñanza en su conjunto, incluyendo el debate acerca de la necesidad de una red nacional de escuelas e instituciones de educación. Se asumía entonces que la escuela no era necesaria para niños que hubiesen crecido en familias decentes, respetables y funcionales, las cuales pudieran dar a los niños todo lo que se espera de una familia decente: desde hornear el pan y organizar la enseñanza hasta realizar procedimientos quirúrgicos menores como el aborto. Sólo si las familias funcionasen mal, se podía exponer a los niños, en caso de necesidad, a los peligros morales y las barbaridades de la vida escolar. Por lo menos hasta finales del siglo XIX se aceptó la idea que la asistencia del niño a la escuela podía desacreditar a la familia. La asistencia a la escuela indicaba que los padres y otros miembros de la familia no habían estado a la altura de sus deberes y dejaban éstos a cargo de otras personas. El 'buen' progenitor no era el que enviaba a su hijo o hija a la escuela, sino aquel que no lo hacía (Smelser 1990). ${ }^{4}$

Se desaconsejó a los padres, por tanto, enviar a sus hijos a la escuela. Las instituciones escolares que intentaron activamente reclutar niños (p.ej. con ciertas ventajas financieras o materiales) fueron criticadas de manera explícita -incluso por filántropos y reformadores sociales. Los padres estaban en mejores condiciones:

to train up their children as olive plants round the domestic table, and rear them within affections towards parents, brothers, sisters and smiling babes, which, for man's good in this life, and the wellbeing of society are worth more than all the Greek and Roman love (Guthrie 1860/1973: 3).

\footnotetext{
${ }^{4}$ Véase, por ejemplo, el esbozo autobiográfico de su infancia que Hugh Millar publicó bajo el título My Schools and Schoolmasters. A cargo de su enseñanza estuvieron sus tíos, por lo que fue educado junto a sus primos. La enseñanza religiosa estuvo a cargo de su mismo tío, si bien también las escuelas dominicales se hacían cargo de esta tarea. "But Sabbath schools my uncles regarded as merely compensatory institutions, highly creditable to the teachers, but very discreditable indeed to the parents and the relatives of the taught; and so they of course never thought of sending us there" (Millar 1865: 37). Ideas muy similares acerca de la educación había formulado John Locke (1878) ya a fines del siglo XVII.
} 
En función de los ideales pedagógicos de este periodo, puede afirmarse sin mayores problemas que las escuelas básicamente eran un síntoma del fracaso de la familia. Su necesidad se debía exclusivamente al hecho de que ciertos padres no estaban a la altura de sus tareas o que algunos niños resultaban ser especialmente rebeldes y desobedientes. Se recomendaba a los padres educar y enseñar a sus hijos en casa. La escuela se consideraba "a general infirmary for mental disease, to which all desperate subjects are sent, as a last resource", como afirmaban los Edgeworths (1801/1992) en el libro Practical Education, todavía reconocido como "the most outstanding treatise on education in circulation during the first half of the nineteenth century" (Curtis \& Boultwood 1962: 111).

En la estela de Jean-Jacques Rousseau, cuyo Emile vio la luz en 1762, se publicaron una serie de tratados y estudios pedagógicos, así como también publicaciones concentradas en la educación de los niños por parte de sus propios padres. Se remarcaba especialmente que la educación natural merecía más atención -y no sólo aquella que tenía lugar en una naturaleza idílica (como en Rousseau), sino también la educación dada por los educadores naturales, los padres (el Emile de Rousseau era huérfano). En varias publicaciones se tomaba posición simultáneamente en contra de la civilización y de la cultura, mientras los métodos de enseñanza escolar eran presentados como símbolos de la cultura par excellence. Así, David Williams (1774: 103), entre otros, buscaba en su Treatise on Education una época en que los seres humanos "(would be) capable of presiding over their own families and educating their children; and render schools themselves unnecessary". En el mismo año, Willem Emery de Perponcher (1774) describió este ideal en sus Instructions d'e un pére a un fils (donde, por cierto, el hijo tenía el significativo nombre de Emile). Algunos años más tarde, este autor escribiría un trabajo de varios tomos sobre la educación privada, mucho más orientado hacia la praxis educativa; el libro se llamó Onderwijz voor Kinderen (1792) (i.e. Lecciones para niños) y tuvo gran influencia en los Países Bajos. ${ }^{5}$

A pesar de esta obvia preferencia por la familia, aparecieron también formas mixtas de educación familiar y escolar. Algunos autores recomendaron sistemas de enseñanza privada en casa, a los que se pudiese atraer también a otros jóvenes y, de esta forma, posibilitar experiencias sociales, imitación y competición. Tempranamente se encuentran ejemplos de esto en la literatura (p.ej. Mulcaster 1581). En la segunda mitad del siglo XVIII,

\footnotetext{
${ }^{5}$ En los años siguientes a la publicación del Emile varios padres intentaron efectivamente educar a sus hijos en base a los principios de Rousseau. Acerca de los resultados las opiniones siguen divididas (véase a este respecto Douthwaite 2002; Baggerman \& Dekker 2005). De todas formas, debe recordarse también que la infancia de Herbert Spencer estuvo marcada por este tipo de experimentos (véase Spencer 1904). En cuanto a la recepción de Rousseau en Alemania, no puede dejar de mencionarse la importancia de la pedagogía filantrópica. Una traducción abundantemente comentada del Emile apareció en los últimos volúmenes de la Enciclopedia Allgemeine Revision des gesamten Schul- und Erziehungswesens [Revisión general del conjunto del sistema escolar y de educación, 1785-1792]. Probablemente para la praxis educativa fueron más significativas las publicaciones y las fundaciones de escuelas de los filántropos que los tratados filosóficos de Rousseau.
} 
Familia y escuela: Algunas reflexiones sobre la diferenciación interna del sistema de la educación

estas voces aparecen con más frecuencia. Esta era la recomendación, por ejemplo, de Joseph Priestley

A number of gentlemen of fortune, whose sons are nearly of an equal age, and free from all tincture of vice, and are all provided with separate tutors [should] contrive to bring them often together, so as to perform certain exercises in common (Priestley 1788/1995: 75).

Aquí se pone de manifiesto que la nobleza era consciente del riesgo de organizar su autoexclusión de la sociedad a través de la educación privada. Sumado a ello, en la transición a la sociedad moderna era fácil darse cuenta que, junto al estamento, ${ }^{6}$ había también una ocupación que había sido elegida y no era necesariamente heredada del padre. Los mismos padres, completamente absorbidos por su vida profesional, apenas disponían de tiempo para la educación de sus hijos. En este contexto, familia y escuela, así como educación privada y pública, aparecieron como alternativas cada vez menos disyuntivas o como mundos opuestos y amenazantes el uno para el otro (Stichweh 1991; Vanderstraeten 2004).

\section{LA DIFUSIÓN DE LA EDUCACIÓN ESCOLAR}

La Europa de la Baja Edad Media y de la modernidad temprana puede ser vista como una sociedad articulada en estamentos. En el plano de su diferenciación primaria, esta sociedad se componía de subsistemas compuestos por grupos estamentales jerárquicamente separados los unos de los otros, a los cuales individuos y hogares pertenecían con la totalidad de sus formas de vida. La ordenación por rangos determinaba las relaciones entre los grupos, es decir, las diferencias relevantes. Familia y hogar eran considerados como el hábitat natural de cada individuo. En el siglo XIX todavía se apela a esto:

God never made men to be reared in flocks, but in families. Man is not a gregarious animal, other than that he herds together with his race in towns, a congeries of families. Born, as he is, with domestic affections, whatever interferes with their free play is an evil to be shunned, and, in its moral and physical results, to be dreaded. God framed and fitted man to grow up... (under) the domestic roof (Guthrie 1860/1973: 2ss).

La familia era idealmente una unidad autárquica e independiente en la que la educación también debía tener cabida. La misma casa parecía, a primera vista, el contexto ideal para proporcionar todo aquello que era necesario para su propia conservación. Todo lo que fuese importante para su

\footnotetext{
${ }^{6}$ NT: La traducción tradicional de Stand es clase. En la obra de Luhmann, sin embargo, el concepto de clase tiene otro término (sozialen Klasse) y una posición definido en su arquitectura teórica. He preferido, en cambio, utilizar el término de estamento que se enlaza mejor con la discusión posterior de los autores acerca de las condiciones de una sociedad estratificada. Esta observación es válida también para las siguientes utilizaciones del término: Stand, Standesbewusstsein, ständisch, etc.
} 
supervivencia y continuación podía proporcionarse de la mejor manera posible dentro de la familia y a través de ella. Del mismo modo, la estructura familiar y la social parecían estar alineadas entre sí. El micro-mundo familiar jerárquicamente estructurado podía preparar adecuadamente a los niños para las relaciones macro-sociales de dominación.

Estas concepciones fueron empero cuestionadas como consecuencia de una serie de profundas transformaciones sociales. A comienzos de la sociedad moderna se levantan nuevas expectativas en torno a la educación y la enseñanza. En la segunda mitad del siglo XVIII tomó impulso la expansión de la escuela y la enseñanza escolar en Europa. En este periodo tuvo lugar un crecimiento explosivo de las publicaciones sobre pedagogía (usualmente como reacción a la publicación del Emile en 1762). En los Países Bajos, por ejemplo, aparecieron en la sexta década del siglo XVIII más tratados de pedagogía que en los 100 años anteriores. Este remarcable aumento de las publicaciones fue acompañado de un cambio en los puntos de partida del debate acerca de (del sitio de) la educación. Asimismo, como consecuencia de la rápida difusión de la escuela primaria, los tradicionales ideales pedagógicos y sociales fueron objeto de una presión cada vez más intensa.

Ello es resultado, entre otros factores, de una crítica a la forma en que los padres cumplen con sus tareas educativas. Igualmente cierto es que quién apoya el ideal de la educación privada en casa de los padres tiene frecuentemente apenas confianza en las habilidades reales de éstos. La escuela se presenta a veces como la segunda mejor opción, como una opción sensata, precisamente porque los padres han descuidado sus deberes educativos. Así lo señala, por ejemplo, Clara Reeve:

If all mothers fulfilled their duties, there would be little occasion for boarding schools; but if they give up their time and attention to dress, to visiting, to cards, to public places, it is better than the children should go to schools, than that they should converse with servants, or play in the streets (Reeve 1792/1974: 73)

En una competición de un club holandés en 1793 (Provinciaal Utrechtsch Genootschap van Kunsten en Wetenschafppen), cuyas reglas fueron vagamente formuladas, se sondearon también los límites entre ambas formas de educación: "¿Qué tipo de educación se debe favorecer? ¿Una pública o una privada? ¿Cuáles son las ventajas y las debilidades de ambas? ¿Existe alguna forma de educación que tenga las ventajas de ambas, pero ninguna de sus desventajas?". En una de las presentaciones premiadas, esta posición se formulaba de la siguiente manera

La educación se debe dar en casa, bien por los mismos padres o bajo su vigilancia. Sin embargo, frecuentemente se da sin su vigilancia y sin recibir su atención, ya sea a través de sirvientas, cuidadores, tutores o tutoras, ellos mismos necesitados aún de educación (Vatebender 1801: 3) 
Familia y escuela: Algunas reflexiones sobre la diferenciación interna del sistema de la educación

La educación en las escuelas se defiende a través de una crítica a las deficiencias y limitaciones de la educación privada. Las otras presentaciones que fueron premiadas y publicadas no tenían una opinión diferente respecto de este tema.

Esta observación sobre la incompetencia pedagógica de los padres se complementa con las crecientes expectativas sociales sobre la educación y la enseñanza. Los avances políticos y económicos de la época facilitaron la realización de una red nacional de instituciones escolares. Así, Niemeyer (1796/1970: 15) escribía en 1796 en Grundsätzte der Erziehung und des Unterrichts für Eltern: " "Desde que se distingue entre educación familiar y educación pública y se ponen ambas en práctica, se discute acerca de si una es mejor que la otra. No faltan razones para ambas posiciones". La autoridad pedagógica y el entusiasmo del profesor por su trabajo, por ejemplo, son temas que ya en el siglo XVIII marcaron fuertemente el debate acerca de las ventajas y desventajas de la educación escolar comparada con la educación familiar. De este modo, se discutió sobre problemas de autoridad y confianza pedagógica, derivados de la mayor singularidad e inexcusabilidad de los errores del profesor en comparación con los de los padres (Ehlers 1766: 165ss. y 213ss.). A finales de siglo, la escuela se vuelve tan marcadamente el centro de todas las reflexiones sobre educación y reforma que la pedagogía se concentra cada vez más en la enseñanza escolar. Entonces se afirma y presupone que el pedagogo o el profesor, carente de las bases de autoridad 'naturales' de los padres, puede reemplazarlas mediante su competencia profesional (Trap 1977).

La resistencia a la escolarización no debe subestimarse -especialmente en países en los que se cultivaba y cultiva fuertemente la conciencia de pertenencia a un estamento. Los happy few que invocaban la validez de la tradición del propio hogar eran aun numerosos en la Inglaterra del siglo XIX. Una parte no menor de los niños de los 'mejores círculos' se preparó durante este siglo para la universidad o la vida laboral en el hogar paterno. De acuerdo con las estadísticas publicadas por las comisiones oficiales inglesas, en 1868 un 11,6\% de los estudiantes matriculados en las universidades de Oxford y Cambridge habían recibido educación solamente en casa de sus padres antes de ingresar. Treinta años después todavía un 11,4\% de quienes asistían a las escuelas secundarias habían sido enseñados previamente solamente en casa de sus padres (véase Hans 1951; Musgrove 1966: 16ss.; Smelser 1991: 39ss.). Para los antes mencionados happy few ingleses, las llamadas "public schools" (Eton, Winchester, etc.) reemplazaron posteriormente a la familia como el ambiente educativo principal, aunque no se redujo en absoluto con esto la sensibilidad por las diferencias de rango.

La implementación de la educación escolar obligatoria es un fenómeno típico del siglo XIX. La legislación correspondiente "[is] adopted in virtually every Western European country in the 'long' nineteenth century,

\footnotetext{
${ }^{7}$ Principios de la educación y de la enseñanza para padres, tutores y educadores.
} 
From Prussia (1763) to Belgium (1914)" (Ramirez \& Boli 1987: 3). Mediante esta legislación se universaliza la asistencia a la educación escolar. ${ }^{8}$ En principio, todos tienen igual acceso a la educación y la enseñanza. A la vista de lo expuesto, la implementación de la obligatoriedad de la educación escolar se puede ver como una especificación de los postulados de inclusión universal que fueron simbolizados, entre otras formas, en los ideales de la Revolución Francesa (Libertad, Igualdad, Fraternidad). Gracias a esta universalización de la educación escolar como obligatoria, se garantizó la inclusión de toda la población en el sistema de la educación (Luhmann \& Schorr 1988: 29ss). En lo que respecta al poder de penetración de los postulados de inclusión en la sociedad moderna funcionalmente diferenciada, la escuela aparece como un ejemplo clásico. Esto no implica, sin embargo, que las diferencias de rango pierdan su poder. ${ }^{9}$ Los postulados de inclusión de la sociedad moderna no van acompañados de posibilidades de realización (Vanderstraeten 2006).

Niklas Luhmann y Karl-Eberhard Schorr han relacionado la tematización de la inclusión en el sistema de la educación con el llamado descubrimiento del niño:

And not least of all, by using the example of the child, the universal inclusion of the larger population in the process of education becomes explainable, because, after all, everyone comes into the world being helpless, everybody grows up as a child, everybody is somehow educated through his environment, and the only question is: how well (Luhmann \& Schorr 1988: 33). ${ }^{10}$

El descubrimiento del niño seguramente favoreció los intereses de los pedagogos (Ariès 1960; Heywood 2001; Wahrman 2004). Conceptos como "naturaleza", "predisposición", "talento" y "esfuerzo" aparecen por primera vez en este contexto y designan una disposición individual que puede neutralizar, al menos en algunos casos, la jerarquía social de los estamentos (La Vopa 1988; Stichweh 1991). De acuerdo con esta idea, todas aquellas restricciones a la educación que no sean atribuibles a ella misma sino por el contrario a su entorno estamental dejan de ser acepta-

\footnotetext{
${ }^{8}$ Para complementar: en la mayoría de los países de los países de Europa no existe obligatoriedad de asistir a la escuela (Schulpflicht) sino obligatoriedad de aprender (Lernpflicht). Esto significa que los padres todavía tienen la posibilidad de educar a sus hijos en su propio hogar. Los padres pueden ocuparse ellos mismos de los aspectos esenciales de la educación o pueden contratarse profesores particulares que se ocupen de las tareas educativas. Actualmente se observa un renovado interés, especialmente en Estados Unidos, acerca de la "home schooling" (para una visión general, véase Bauman 2001).

${ }^{9}$ Un tema mencionado frecuentemente a finales del siglo XVIII. Así opina, por ejemplo, Peter Villaume que el educador "no [debe] hacer a su alumno más perfecto de los que su estamento lo permita; excepto cuando él vea en su esfuerzo que éste pertenece a un estamento diferente" (Villaume 1785: 526; muy similar, Wolff 1780/1977:107s.). También para Condorcet la educación de alguien estaba determinada por su posición en la jerarquía de los estamentos. "Les enfants, suivant la riquesse de leurs parents, les circonstances ou se trouvent leurs familles, l'état auquel on les destine, peuvent donner plus ou moins de temps a l'instruction" (Condorcet 1792/1847: 188s.).

${ }^{10}$ NT: Citado de acuerdo a la traducción en inglés, si bien se mantiene la referencia al libro original en alemán. Véase Luhmann, N. \& Schorr, K.-E. (2000). Problems of Reflection in the System of Education (translated by Rebecca A. Neuwirth), Münster and New York: Waxmann, p. 39.
} 
Familia y escuela: Algunas reflexiones sobre la diferenciación interna del sistema de la educación

das. En otras palabras: los sistemas sociales se conectan de manera circular con su entorno. El concepto de enactment de Karl Weick (1979) describe el proceso de creación de un entorno en el sistema que después da forma a su entorno relevante. Así, los nuevos constructos imponen una perspectiva educativa a la realidad, la que después requiere de intervenciones educativas.

La nueva valoración del niño fue acompañada de una redescription de los roles complementarios. Ya señalamos que aumentaron las expectativas sobre los padres -especialmente hacia las madres. En el siglo XVIII tuvo lugar una sensibilización de los educadores naturales hacia la pedagogía (Gilgenmann 1994). Simultáneamente comenzó la discusión acerca de los practicantes formados profesionalmente (maestros de escuela). Así, Ernst Christian Trapp afirmaba:

Aquello que se debe realizar con cuidado, debe haber sido también preparado adecuadamente; se debe ser experto en los principios y haber aprendido su aplicación tanto teórica como práctica. Con otras palabras, esto significa: la educación, en tanto arte con características peculiares, debe ser practicada por sus propias personas (Trapp 1977: 21)

La insuficiencia de la educación natural da fundamentos a la necesidad social de la educación, a la que el pedagogo puede apelar al hacer demandas o justificarse. Ella funda la profesionalidad de la praxis de la enseñanza y la necesidad de una preparación especializada. El mismo Trapp fue nombrado en 1779 catedrático de pedagogía (jel primero del mundo!) -cuyo nombramiento tuvo lugar obviamente en Halle.

Especialmente desde mediados del siglo XIX tuvo lugar una revalorización de la profesión docente. Dicha labor se considera cada vez más frecuentemente como una ocupación plenamente válida y no sólo como una actividad complementaria que puede compatibilizarse con otras (agricultor, sacristán, etc.). Otros roles adicionales también pudieron adquirir formas estables en esta marea de cambios. Se consolidan las normas, administración y supervisión de las escuelas. Se les otorga más atención a los planes de estudio y los currículos. En el transcurso del siglo XIX surgen redes extensas y organizadas de escuelas e instituciones de educación; desde ese entonces la existencia de estas redes se ha convertido en algo dado por hecho. Y sólo después de que la educación escolar diferenciada como foco de todos los avances se convierte en el centro de interés de la pedagogía, la educación familiar puede repensarse en lo que respecta a su relación con la escuela. En cualquier caso, con la inclusión de toda la población en estas instituciones, la doble vida (temporal y biográfica) de los escolares en la familia y la escuela se convirtió en una experiencia social generalizada.

La mera contraposición entre ambos ambientes empero sobrevivió. De este modo, se observan recíprocamente familia y escuela, en aspectos específicamente pedagógicos, aun como ámbitos estructuralmente desca- 
lificables. La diferencia relativa a la competencia profesional entre profesores y padres se hace siempre en detrimento de estos últimos. Estos son vistos como legos en pedagogía y necesitados de formación, y el programa correspondiente se llama educación de los padres. $\mathrm{O}$ bien: los rendimientos de socialización de la familia son deficitarios y necesitan compensarse dadas las exigencias de la escuela. Por otra parte, en lo que respecta a la familia, persisten los resentimientos contra la escuela como organización. Ella resulta descalificable fácilmente como un lugar frío, carente de cariño e incluso inhumano, en el que un montón de niños son continuamente evaluados, medidos, censurados y seleccionados por parte de los profesores. Los escolares tampoco están siempre felices en la escuela: "La clase permanece relativamente monótona; los estudiantes observan con gratitud cuando accidentalmente uno de sus compañeros se cae de la silla" (Markowitz 1981: 92.). Es claro que la pedagogía puede sacar pocos dividendos del dualismo estructural entre familia y escuela. Hace falta, en su mayor parte, una reflexión consolidada sobre las formas de división de tareas entre ambos ambientes educativos y las relaciones que mantienen entre sí. La pedagogía fracasa aquí como "ciencia del cuidado del sistema". Con todo, la diferenciación entre familia y escuela tampoco ha sido analizada en detalle en el ámbito de la sociología.

\section{LA DIFERENCIACIÓN ENTRE FAMILIA Y ESCUELA}

La diferenciación y separación entre familia y escuela tiene un evidente paralelo con otro resultado de la diferenciación especialmente importante para la sociedad moderna: la separación entre hogar y trabajo, entre vida familiar y laboral. En la literatura se usa esta analogía para presentar la relación entre familia y escuela. Se habla entonces explícitamente de la separación entre un espacio vital y uno escolar como un atributo característico de la modernidad (Fend 1980: 115ss., 2006). Ello se puede mantener en general todavía. De todas formas, es válido también acá destacar los límites de esta analogía, en tanto dicha designación lleva inmediatamente a la problemática de la interacción entre el hogar paternal y la escuela. La "separación de los roles laborales y familiares", mediada contractual y monetariamente (Parsons 1972: 100), se encuentra diferenciada de manera más definida que la separación entre los roles familiares y escolares: las escuelas no pueden neutralizar e ignorar el contexto familiar de sus estudiantes, especialmente de sus padres, al menos no en el mismo grado en que las empresas pueden hacerlo con su personal. De una manera $u$ otra, las escuelas deben aceptar a los padres como un grupo de referencia legítimo en su entorno. Los profesores pueden difícilmente rehusarse si los padres se presentan en defensa de sus hijos en la escuela: los días de consulta de padres y profesores, así como también las reuniones de apoderados están institucionalizadas como ofertas de interacción escolar (Maclure \& Walker 2000). En ocasiones también los profesores, cuando tienen problemas en particular con algunos estudiantes, se remi- 
Familia y escuela: Algunas reflexiones sobre la diferenciación interna del sistema de la educación

ten directamente a los padres de éstos y los ponen bajo su control si es que ellos no pudieron lograr nada. No existe nada similar en la relación entre trabajo y familia. El trazado de límites y la separación entre sistema y entorno parecen estar considerablemente más 'distanciados'.

En un sentido similar, Talcott Parsons describe la dinámica propia entre familia y escuela como la de sistemas parciales socialmente diferenciados. En su análisis de la familia americana tradicional, Parsons escribe: "But looked at as a part of the society, the family is, even in primitive societies, a specialized, i.e., differentiated, part of a larger system; it is quite erroneous to regard it as a 'microcosm' of the whole" (Parsons 1955: 33). En el análisis de la universidad americana esto significa: "Although socialization in higher educational institutions helps prepare for citizenship roles in a societal community which emphasizes institutionalized individualism, the academic community is not a microcosm of the society but a differentiated part of the society" (Parsons \& Platt 1973: 203). Nos ceñiremos a este punto de partida (Dreeben 1968). Las reflexiones siguientes y finales buscan abordar precisamente la separación entre familia y escuela sobre este trasfondo. El objetivo es explicar de una manera relativamente descriptiva lo que esto significa en términos estructurales y la manera en que se presenta fácticamente en el contexto de la experiencia de los escolares. Esta tarea será abordada brevemente, y sin pretensiones de exhaustividad, en cinco puntos (a-e).

(a) La diferenciación entre familia y escuela es evidente inicialmente en términos espaciales: vida familiar y enseñanza escolar tienen lugar en sitios distintos. También desde la perspectiva de la familia (y de manera análoga al trabajo profesional), los eventos de la escuela ocurren fuera de la casa. De esta forma, 'estar en la escuela' significa por lo tanto para el niño 'no estar en la casa'. Con esto se presupone que vida familiar y clase escolar se localizan en sitios específicos y diferentes el uno del otro: acá el niño vive y allá asiste a la escuela. Una consecuencia es que el niño debe recorrer diariamente un camino de ida y de vuelta a la escuela y se mueve en un espacio intermedio. Padres y profesores tienen frecuentemente dificultades para controlar dicho espacio. Los escolares, por su parte, aprovechan allí sus ocasiones: en ese espacio se fuma el primer cigarrillo, se ve al primer novio o la primera novia, o a los otros amigos, etc.

Adicionalmente, la estructura espacial y arquitectónica de la sala de clases expone casi irremediablemente a los profesores a una intensa observación por parte de sus alumnos. Cada estudiante puede tener toda la atención del profesor sólo de manera excepcional, sin embargo, todos deben permanecer atentos y tienen, de esta manera, suficientes ocasiones para ver cómo el profesor se ocupa de la clase o de otros estudiantes. De este modo, por mucho que los maestros intenten distanciarse, no pueden evitar estar expuestos a la mirada escrutiñadora de sus alumnos. Como se puede comprobar fácilmente; los profesores son observados despiadadamente como personas normales, demasiado normales. Sus debilidades y 
peculiaridades son observadas y aprovechadas como un gratificante tema común de conversación entre los estudiantes.

(b) La siguiente diferenciación entre familia y escuela es temporal. El niño asiste diariamente a la escuela a ciertas horas y de acuerdo con una planificación horaria determinada. Es posible aplicar explícitamente aquí la analogía con las jornadas diarias de trabajo. La escuela también tiene un claro sentido temporal que se orienta de acuerdo con límites temporales $\mathrm{y}$, por consiguiente, a través de una separación temporal de sistemas. La escuela o, mejor dicho, la clase, es algo que comienza y termina diariamente en un momento preciso. Cuando la escuela acaba, los niños modifican su comportamiento repentina y drásticamente: el antes y el después de la hora de cierre de la escuela o de la finalización de la clase es, sin que se necesite haber escuchado el timbre, inmediatamente visible en el comportamiento de los estudiantes. No es necesario siquiera mencionar cuán artificial o improbable es esta organización temporal, con su precisa demarcación sistémica basada en la hora y, precisamente por ello, cuánto ha de serlo para los niños. Más sorprendente aún es ver, sin embargo, cuán (relativamente) fácil es para los niños que llevan un reloj adquirir la conducta temporal adecuada y habituarse a ella como una conducta normal -si bien desde el punto de vista del profesor algunos "simplemente no quieren aprender" a ser puntuales.

Las familias, por su parte, se han adaptado internamente de manera no menos sorprendente a la organización escolar del tiempo, especialmente a los límites que les son dados desde ahí, es decir, externamente. Salvo en el periodo de vacaciones, una organización familiar de la vida cotidiana apropiada para la escuela le asegura a esta última que el niño sea enviado a clases bien preparado y puntualmente; el sistema escolar de medio día permite así que millones de amas de casa y madres se preocupen que sus hijos almuercen después de clases. Por otra parte, las vacaciones pueden obligar a las familias a idear complicadas soluciones provisionales para las que deben llamar a abuelos, amigos, vecinos, etc.

(c) La separación entre familia y escuela exige de los niños además distinguir claramente entre referencias sistémicas. Por una parte, el estudiante debe saber manejar la diferencia de sentido entre las formas de comportamiento que pertenecen a la familia y que son adecuadas en el hogar, y aquellas que son correctas o necesarias (frente al profesor y los compañeros de clase) en la escuela. Por otra parte, el niño debe construir su comportamiento de tal manera de tener a su disposición el comportamiento adecuado en el lugar y momento correctos, y también de modo tal que no cometa equivocaciones como jugar, llamar a su madre en clases o levantar la mano y pedir la palabra en casa, con lo que quedaría en ridículo. Para que la diferenciación entre escuela y familia pueda operar adecuadamente, se necesita de práctica y de una distinción estable entre formas heterogéneas de comportamiento mediante las cuales también ter- 
Familia y escuela: Algunas reflexiones sobre la diferenciación interna del sistema de la educación

ceros sean capaces de reconocer la diferencia sistémica (familia o escuela) aplicable. ${ }^{11}$

En lo que respecta a la diferencia decisiva entre familia y escuela para el niño, es fundamental que ambos órdenes heterogéneos de comportamiento no se mezclen y que se realicen en dos sitios sociales distintos, de modo que sea posible concentrarse en cada uno de ellos en términos temporales y especiales: la escuela es sólo, o primariamente, el sitio de la enseñanza, de la interacción profesor-alumno; por el contrario, la vida familiar y también el trabajo del padre pertenecen a otra parte. Correspondientemente, los eventos de la familia (si bien atravesados por extraños al sistema como las tareas escolares) se separan en un sitio familiarprivado en el que otras cosas, como la enseñanza escolar, no tienen cabida. En cada localización, las referencias sistémicas de la acción son claramente identificables y, en lo que respecta a la conciencia de los límites, diferenciables entre sí.

(d) Para los niños, la separación institucional entre familia y escuela se plasma también en que ellos van 'solos' y sin padres a la escuela, y en que después de la escuela retornan a sus familias sin profesores ni compañeros de clase. Que los niños deban desenvolverse normalmente en la escuela 'sin padres' y distanciados de su entorno familiar significa, sobre todo, que en la escuela los padres están ausentes y no se encuentran cerca para interactuar. Los escolares interactúan con profesores y compañeros de clase en la escuela sin la protección ni el respaldo de sus padres presentes; ellos deben arreglárselas por sí mismos, cuestión que al inicio de la carrera escolar puede ser muy dolorosa. Pero también la ausencia de los padres significa la supresión de su vigilancia y control; la escuela tiene entonces, por así decirlo, también una dimensión de libertad. Es también importante que la presencia excepcional de los padres en la escuela (sobre todo en la sala de clases) normalmente libera al profesor de la necesidad de una atención continua al entorno familiar del niño.

De ello resulta que la separación entre familia y escuela tiene un aspecto que refiere a la información, precisamente porque los padres no asisten todos los días a la escuela de sus hijos y, por eso, los eventos de la escuela no son para ellos transparentes. Ambas partes presentan un déficit estructural de información recíproca. La distancia informativa entre la casa paterna y la escuela tiene muchas consecuencias (Tyrell 1982), aunque también facilita a los escolares la mentira y el encubrimiento. Así, los padres no pueden valorar usualmente cuán difícil serán las próximas

\footnotetext{
${ }^{11}$ Las escuelas y los profesores deben lidiar también con destinatarios críticos, precisamente porque los niños tienen que ir a la escuela. Así, los estudiantes suelen entretenerse observando el comportamiento de su maestro y buscando errores, debilidades, etc. Ellos observan si el profesor los está observando o si momentáneamente se encuentran fuera de su área de control visual; intentan esconderse detrás de sus compañeros de clase, simulan escuchar atentamente; hacen gestos como si hubiesen entendido todo, etc. Aquel estudiante que insista en mantener su individualidad e independencia encuentra en el contexto escolar y en la presencia de sus compañeros oportunidades permanentes para no participar-y para demostrar la elección de esta alternativa.
} 
pruebas o exámenes de los estudiantes. El niño que busque escapar del inminente paseo del domingo puede sacar ventaja de este déficit de información de los padres. De manera similar, los profesores son incapaces de juzgar la veracidad de los problemas familiares o de tráfico a los que los estudiantes aluden cuando llegan tarde nuevamente. La diferenciación de ambos sistemas parciales -educación y familia- lleva aparejada también una socialización de los niños en esta dirección.

(e) La intransparencia de los eventos de la escuela y la incapacidad de dirigirlos desde la casa de los padres tiene otra dimensión, generalmente pasada por alto, que no es ni puede ser menos frágil. Se trata de la dinámica propia de la relación de los escolares entre sí, apenas gobernable por padres o profesores, y de la formación de grupos y subculturas (principalmente) entre compañeros de clase. Sin duda, las clases comienzan la mayoría de las veces en un punto cero social: los estudiantes no se conocen entre sí o, si es que lo hacen, es sólo en ciertos aspectos específicos. De inmediato, sin embargo, comienzan dinámicas de grupo, casi siempre de manera automática y a gran velocidad, las cuales se normalizan mediante las preferencias de relación de los estudiantes, entre otros factores.

Es claro que ni padres ni profesores pueden controlar estos procesos. Los padres tienen apenas conocimiento acerca de las amistades de su hijo en la sala de clases, a cuál pandilla se une, con quién tiene relaciones amistosas u hostiles, qué hace con sus amigos en el recreo, etc. A los ojos de los padres, bien podrían ser en realidad los amigos indeseables. Que el clima de la clase pueda más bien desarrollarse, para decepción de los padres que valorizan el rendimiento académico, hacia la apatía en torno al aprendizaje y a la entrega de premios y a la premiación y adquisición de prestigio en base a abundantes actividades extraescolares, es algo familiar para todo conocedor de la escuela. Lo que se normalice en términos de dinámica de grupos es difícilmente modificable y apenas reversible; en términos generales, la cooperación forzosa entre padres y profesores puede hacer poco en contra de esto.

\section{PARA FINALIZAR}

En el ámbito de la sociología de la educación existe una importante tradición que hasta ahora se ha interesado principalmente por la escuela. Desde Émile Durkheim (1993: 108-109) se ha descrito a la escuela como un "microcosmos" social, como un microcosmos de la sociedad en la sociedad, cuya función reside en la transmisión de los valores y normas dominantes. Este punto de partida ha llevado a que se dedique una atención especial a la escuela y a la correspondencia entre escuela y sociedad. Así, las demandas que se hacen sobre la conducta en las situaciones de enseñanza son vistas como representativas de las demandas presentes en la vida cotidiana de la sociedad moderna -y esto no sólo en el nivel del currículo oficial, sino también en el de las estructuras ocultas o latentes de una modernidad orientada hacia el logro. En esto se tiene la impresión de 
Familia y escuela: Algunas reflexiones sobre la diferenciación interna del sistema de la educación

que la sociología de la educación se basa todavía en la idea de una mano invisible que puede asegurar la integración social. No obstante, estos puntos de partida suelen tener también por consecuencia que la diferenciación interna del sistema de la educación generalmente cae o permanece fuera del campo de atención de la sociología de la educación.

Los análisis aquí presentados no ofrecen un resultado definitivo relativo a las relaciones entre familia y escuela. Hemos visto dicha relación como diferenciación interna del sistema de la educación y la hemos analizado en el contexto de la diferenciación de dicho sistema funcional. No intentamos con ello hacer un mero re-etiquetado. Más bien se busca introducir la relación entre familia y escuela en el contexto de la teoría de la diferenciación funcional para, de esta manera, poder formular preguntas y obtener puntos de vista que superen el estado actual de la discusión y que, además, permitan arrojar luces sobre las ya mencionadas dificultades pedagógicas de la materia. Para ello, hemos inspeccionado el equipamiento analítico y medios de reflexión que ofrece la teoría de la diferenciación funcional en aras de una adecuada conceptualización de las relaciones entre familia y escuela. En nuestra opinión, se pueden obtener importantes avances sociológicos mediante esta aproximación. Dichos avances pueden ser un factor decisivo que permita conectar la tradición teórica de la disciplina con datos históricos y empíricos.RM

\section{REFERENCIAS}

Ariès, P. (1960). L'enfant et la vie familiale sous l'Ancien Régime. Paris: Plon.

Bauman, K.J. (2001). Home Schooling in the United States: Trends and Characteristics. Washington DC: US Census Bureau (Population Division Working Paper Series no. 53).

Baggerman, A. \& Dekker, R. (2005). Kind van de toekomst. De wondere wereld van Otto van Eck (1780-1798). Amsterdam: Wereldbibliotheek.

Condorcet (1847). Sur l'instruction publique. In Euvres, Tome 7 (pp. 167-573). Paris: Didot.

Curtis, S.J. \& Boultwood, M.E.A. (1962). An Introductory History of English Education since 1800. London: University Tutorial Press.

De Perponcher, W.E. (1774). Instructions d'un père à son fils. Utrecht: Spruit.

De Perponcher, W.E. (1782). Onderwijs voor kinderen, 3 vols. Utrecht: Schoonhoven.

Douthwaite, J.V. (2002). The Wild Girl, Natural Man, and the Monster. Dangerous Experiments in the Age of Enlightenment. Chicago: University of Chicago Press.

Dreeben, R. (1968). On What is Learned in School. Reading: Addison-Wesley.

Durkheim, É. (1993). Éducation et sociologie. Paris: Quadrige-PUF.

Edgeworth, R. \& Edgeworth, M. (1801/1992). Practical Education. London: Routledge.

Ehlers, M. (1766). Gedanken von den zur Verbesserung der Schulen nothwendigen Erfordernissen. Altona/Lübeck: Iversen.

Fend, H. (1980). Theorie der Schule. München: Urban-Schwarzenberg.

Fend, H. (2006). Neue Theorie der Schule. Wiesbaden: VS Verlag für Sozialwissenschaften. 
Gilgenmann, K. (1994). Die Familie als Erziehungsgemeinschaft. Zur Beschreibung der Funktion von Familien in der modernen Gesellschaft. System Familie 7, 212228.

Guthrie, T. (1860/1973). Seed-time and Harvest of Ragged Schools. Montclair N.J.: Patterson Smith. Available in www.newble.co.uk/guthrie/plea1.html

Hans, N. (1951). New Trends in Education in the Eighteenth Century. London: Routledge \& Kegan Paul.

Heywood, C. (2001). A History of Childhood: Children and Childhood in the West from Medieval to Modern Time. Oxford: Blackwell.

Imelman, J. (1998). Kinderopvang: een discutabel antwoord op een maatschappelijk probleem. In: B. Levering, G. Biesta \& I. Weijers (Eds.), Thema's uit de wijsgerige en historische pedagogiek (pp. 142-147). Utrecht: SWP.

Inkeles, A. \& Sirowy, L. (1983). Convergent and Divergent Trends in National Educational Systems. Social Forces, 62, 303-333.

Kay-Shuttleworth, J. (1862/1993). Four Periods of Public Education. London: Routledge.

La Vopa, A. (1988). Grace, Talent, and Merit: Poor Students, Clerical Careers, and Professional Ideology in Eighteenth-Century Germany. Cambridge: Cambridge University Press.

Locke, J. (1878). Gedachten over opvoeding. Groningen: Versluys.

Luhmann, N. \& Schorr, K.E. (1982). Personale Identität und Möglichkeiten der Erziehung. In: Zwischen Technologie und Selbstreferenz. Fragen an die Pädagogik (pp. 224-261). Frankfurt: Suhrkamp.

Luhmann, N. \& Schorr, K.E. (1988). Reflexionsprobleme im Erziehungssystem. Frankfurt a.M.: Suhrkamp.

Markowitz, J. (1982). Relevanz im Unterricht - eine Modellskizze. In N. Luhmann \& K.E. Schorr (Eds.), Zwischen Technologie und Selbstreferenz. Fragen an die Pädagogik (pp. 87-115). Frankfurt: Suhrkamp.

Maclure, M. \& Walker, B. (2000). Disenchanted Evenings: The Social Organization of Talk in Parent-Teacher Consultations in UK Secondary Schools. British Journal of Sociology of Education, 21: 5-25.

Millar, H. (1865). An Autobiography. My Schools and Schoolmasters; or, The Story of my Education. Boston: Gould \& Lincoln.

Mulcaster, R. (1581). Positions Concerning the Training Up of Children. London: Vautrollier.

Musgrove, F. (1966). The Family, Education and Society. London: Routledge \& Kegan Paul.

Niemeyer, A. (1796/1970). Grundsätze der Erziehung und des Unterrichts für Eltern, Hauslehrer und Erzieher. Paderborn: Schöningh.

Parsons, T. (1955). The American Family: Its Relations to Personality and to the Social Structure. In T. Parsons \& R.F. Bales (Eds.), Family, Socialization and the Interaction Process (pp. 3-33). London: Routledge \& Kegan Paul.

Parsons, T. (1972). Das System moderner Gesellschaften. München: Juventa.

Parsons, T. \& Platt, G.M. (1973). The American University. Cambridge: Harvard University Press.

Priestley, J. (1788/1995). Miscellaneous Observations Relating to Education. Bristol: Thoemmes.

Ramirez, F. \& Boli, J. (1987). The Political Construction of Mass Schooling: European Origins and Worldwide Institutionalization. Sociology of Education, 60: 2-17.

Reeve, C., (1792/1974). Plans of Education. New York: Garland.

Smelser, N.J. (1990). The Contest between Family and Schooling in NineteenthCentury Britain. In J.C. Alexander \& P. Colomy (Eds.), Differentiation Theory and Social Change (pp. 165-186). New York: Columbia University Press. 
Familia y escuela: Algunas reflexiones sobre la diferenciación interna del sistema de la educación

Smelser, N.J. (1991). Social Paralysis and Social Change: British Working-Class Education in the Nineteenth Century. Berkeley: University of California Press.

Spencer, H. (1904). An Autobiography, 2 vols. London: Williams und Norgate.

Stichweh, R. (1991). Der frühmoderne Staat und die europäische Universität. Frankfurt: Suhrkamp.

Trapp, E.C. (1977). Versuch einer Pädagogik. Paderborn: Schöningh.

Tyrell, H. (1982). Familienalltag und Familienumwelt. Überlegungen aus systemtheoretischer Perspektive. Zeitschrift für Sozialisationsforschung und Erziehungssoziologie, 2, 167-188.

Vanderstraeten, R. (2004). The social differentiation of the educational system. Sociology, 38, 255-272.

Vanderstraeten, R. (2006). Soziale Beobachtungsraster: Eine wissenssoziologische Analyse von statistischen Klassifikationsschemata. Zeitschrift für Soziologie, 35, 193-211.

Vatebender, G.C.C. (1801) Antwoord op de Vraage: Welke wijze van Opvoeding is de meest verkiezelijke? In Verhandelingen van het Provinciaal Utrechtsch Genootschap van Kunsten en Wetenschappen, $I X$. Utrecht: Wild \& Altheer

Villaume, P. (1785). Ob und in wie fern bei der Erziehung die Vollkom $\neg$ menheit des einzelnen Menschen seiner Brauchbarkeit aufzuopfern sey? In J.H. Campe (Ed.), Allgemeine Revision des gesammten Schul- und Erziehungswesens von einer Gesellschaft praktischer Erzieher (pp. 435-616). Hamburg: Ernst Bohn.

Wahrman, D. (2004). The making of the modern self: Identity and culture in eighteenth-century England. New Haven: Yale University Press.

Weick, K.E. (1979). The Social Psychology of Organizing. New York: Random House.

Wilderspin, S. (1840): The Infant System. London: Hodson. Available in www.fullbooks.com/The-Infant-System1.html

Williams, D. (1774). A Treatise on Education. London: Payne.

Wolff, B. (1780/1977: Proeve over de opvoeding, aan de Nederlandsche moeders. Meppel: Boom.

SOBRE LOS AUTORES

Antes de su retiro, Hartmann Tyrell, se dsempeñó como profesor de la Facultad de Sociología de la Universidad de Bielefeld (Alemania). Sus área de especialización son teoría social, sociología histórica, sociología de la religión y sociología de la familia. Entre sus publicaciones destacadas se encuentran Soziale und gesellschaftliche Differenzierung. Aufsätze zur soziologischen Theorie (2008) y 'Religion' in der Soziologie Max Webers (2014).

Raf Vanderstraeten es profesor en el Departamento de Sociología de la Universidad de Gante (Bélgica). En el periodo 2016-2017, Vanderstraeten desarrolla actividades académicas como profesor visitante en el Departamento de Sociología de la Universidad de Chicago (USA). Sus áreas de especialización son teoría social, sociología histórica, sociología de la ciencia y sociología de la educación. Entre sus publicaciones destacadas se encuentran Parsons, Luhmann and the theorem of double contingency (2003), Inclusion ideals and inclusion problems: Parsons and Luhmann on religion and secularization (2015) y Reconfiguring organisational patterns of power: An etnography on the functioning of academic credentials in Chile (2015).

\section{CONTACTO}

Universiteit Gent

Faculty of Political and Social Sciences

Sociology

Center for Social Theory 
Hartmann Tyrell \& Raf Vanderstraeten

Korte Meer 3-5, BE-9000 Ghent

Raf.Vanderstraeten@UGent.be

Recibido: septiembre 2016

Aceptado: enero 2017 